\title{
DNA methylation regulates expression of the iron regulatory peptide hepcidin in hepatic cell lines
}

\author{
A. Hussain ${ }^{1}$, R. Clarkson ${ }^{1}$, I. Morison ${ }^{2}$ and P.A. Sharp ${ }^{1}$ \\ ${ }^{1}$ Diabetes \& Nutritional Sciences Division, King's College London, UK and \\ ${ }^{2}$ Department of Pathology, University of Otago, New Zealand
}

Iron homeostasis is maintained by matching duodenal iron absorption to body requirements for haemoglobin synthesis and cellular metabolism. Levels of iron in the body are sensed primarily by hepatocytes, which respond by controlling the production and release of the iron regulatory peptide hepcidin. At the cellular level, hepcidin acts to limit iron release from enterocytes and reticuloendothelial macrophages, thus controlling the delivery of iron to the bone marrow ${ }^{(1)}$. Hepcidin levels are extremely low in population groups with iron deficiency, but elevated in those with high body iron. Interestingly, serum hepcidin concentrations vary significantly within a normal healthy population ${ }^{(2)}$. The reason for this variability is unclear, but we have hypothesised that this may be partly attributable to differences in the epigenome.

Here we have investigated the effects of the DNA de-methylating agent, 5-deoxy-2'-azacytidine (AZA) on expression of $H A M P$, the gene encoding hepcidin. DNA and RNA were isolated from HepG2 and Huh7 cells treated with AZA (5 $\mu$ M, 72 h). RT-PCR was used to quantify changes in HAMP mRNA expression. Samples of DNA were subjected to bisulphite conversion and RT-PCR was used to predict whether specific $\mathrm{CpG}$ sites in the HAMP promoter were methylated or unmethylated. Data were analysed using Student's unpaired t-test; statistical differences of $\mathrm{P}<0.05$ were considered significant.

AZA treatment increased $H A M P$ expression 3.2-fold $(\mathrm{P}<0.01)$ in Huh7 cells, but did not significantly alter $H A M P$ levels in HepG2 cells. Interestingly, baseline expression of $H A M P$ was 24 -fold higher in HepG2 cells than in Huh7 cells (P<0.01). To test whether differences in baseline $H A M P$ levels could be explained by methylation, we carried out PCR analysis of the $H A M P$ promoter following bisulphite conversion. The University of California Santa Cruz Genome Browser database (https://genome.ucsc.edu/) identified 2 CpGs within both the forward and the reverse primer annealing regions. However, there were no differences in levels of PCR amplicons generated in HepG2 and Huh7 cells using combinations of methylation-specific primers.

Our results support the hypothesis that DNA methylation may regulate the expression of HAMP. However, our analysis of HAMP promoter methylation indicates that the effects are not mediated by $4 \mathrm{CpGs}$ clustered around the translation start site.

1. Ganz T (2013) Physiol Rev 93, 1721-1741.

2. Handley S, Couchman L, Sharp P, et al. (2017) Bioanalysis 9, 541-553. 